\title{
BUCHBESPRECHUNGEN
}

\section{DIE NEUORDNUNG DES INTERNATIONALEN SEERECHTS}

\author{
Besprechungsaufsatz \\ Von Herbert Krüger \\ Rudolf Bernhardt (Hrsg.)
}

Die Schiffahrtsfreiheit im gegenwärtigen Völkerrecht -

Berichte einer Studientagung der Deutschen Gesellschaft für Völkerrecht

C. F. Müller, Karlsruhe, 1975

Wolfgang Graf Vitzthum

Der Rechtsstatus des Meeresbodens

Duncker \& Humblot, Berlin, 1972

Evan LUARD

The Control of the Sea-Bed - A New International Issue

Heinemann, London

Jochen EHMER

Der Grundsatz der Freiheit der Meere und das Verbot

der Meeresverschmutzung

Duncker \& Humblot, Berlin, 1974

Renate Platzöder / Wolfgang Graf Vitzthum

Zur Neuordnung des Meeresvölkerrechts auf der Dritten

Seerechtskonferenz der Vereinten Nationen

Stiftung Wissenschaft und Politik, Ebenhausen, 1974

Renate Platzöder

Third United Nations Conference on the Law of the Sea -

Documents of the Caracas Session

Institut für Internationale Angelegenheiten (Werkhefte Bd. 29), Hamburg 1976

A.

Die Zeit scheint nicht mehr allzu fern zu liegen, da das Meer allein noch von der Gnade des Menschen leben wird - so wie es heute für Landschaft und Tierwelt auf dem Festland bereits weithin zutrifft. Bisher war es die Unerschöpflichkeit von Weite, Kräften und Schätzen, die eine Herrschaft des Menschen über die Meere ausschloß und entbehrlich machte. Der explodierende Bedarf nach Rohstoffen in Verbindung mit unerhörter Perfektion der Technik haben es bewirkt, daß selbst die Unerschöpflichkeit des Meeres erschöpft zu werden droht, wenn sie nicht in manchen Bereichen, wie insbesondere im Falle der Fischbestände, bereits weithin erschöpft ist. Knappe Güter aber müssen bewirtschaftet werden, gleichgültig, 
ob diese Knappheit von Hause aus besteht oder vom Menschen geschaffen ist. Insbesondere wenn das Meer als Familiengut der Menschheit („common heritage of mankind“) gewertet wird, dann sind es vor allem auch die zukünftigen Generationen, die nicht durch einen Raubbau an den Schätzen des Meeres vor ein Nichts gestellt werden dürfen.

Es ist die geschichtliche Aufgabe der Dritten Seerechtskonferenz, die Nutzung der Meere angesichts solcher Entwicklungen in geordnete Bahnen zu lenken. Es ist also nicht etwa allein ein freiheitsblinder Hang zum Dirigismus, der hier am Werke ist - wie jene Art bequemer Erklärung unbequemer Phänomene meint, die auf einen „Bösen Mann“ oder „Schlechten Geist“ zu rekurrieren pflegt, obwohl hierdurch zwar moralische Erhebung gewonnen, in der Sache aber nichts gefördert oder auch nur gerettet werden kann. M. a. W.: Es ist auch an dieser Stelle falsch und sinnlos, einen Prozeß der „Freiheitsfeinde“ gegen die „Freiheitsfreunde" aufziehen zu wollen, ihn von vornherein für die Industrieländer verloren $\mathrm{zu}$ geben (vgl. die vielfach $\mathrm{zu}$ findende Wendung, daß sie „die Verlierenden der Seerechtskonferenz" sein werden) und sich dann am Gedanken zu erlaben, $\mathrm{da} ß$ in dieser Welt nun einmal das Gute nicht zu siegen pflegt. Wenn die „Freiheit" ganze Fisch-Populationen ausgerottet hat oder auszurotten droht, wenn die ungeordnete Ausbeutung des Meeresbodens ein Privileg, ja Monopol der zwei, drei stärksten Mächte bewirken würde, wenn ganze Meere so verschmutzt werden, daß jeder andere Gebrauch als der einer Mülldeponie an den Rand gedrängt wird, dann ist es nicht die Freiheit, die gefährdet wird - sie ist es, die gefährdet, und zwar vor allem sich selbst.

Wenn solcher $Z$ wang selbstgeschaffener Tatsachen im Prinzip zu einer „Terranisierung des Meeres " ${ }^{1 "}$ führt, dann sollte man die Freiheit so weit wie möglich dadurch zu wahren suchen, daß die zu schreibende Ordnung der Nutzung der Meere so freiheitlich wie möglich ausfällt. In dieser Hinsicht stellen sich allerdings Alternativen, zwischen denen gewiß nicht leicht $\mathrm{zu}$ entscheiden ist. Die wichtigste scheint mir zu sein die Wahl zwischen den möglichen Subjekten und damit Garanten der Ordnung: Soll es die Völkergemeinschaft sein, soll das Meer also „internationalisiert“ werden? Oder soll man diese Ordnung den einzelnen Staaten anvertrauen, soll das Meer also "nationalisiert" werden? (was auf jeden Fall zunächst einmal den Ausschluß der etwa 50 „land-locked“-Staaten bedeuten würde). In der Praxis fehlt es nicht an Stimmen, die die zweite Lösung vorziehen: Hier setzt man auf konkrete Arrangements, mit denen man besser $\mathrm{zu}$ fahren hofft als unter einer allgemeinen Regelung, die die individuelle Mächtigkeit nicht zum Zuge kommen, vielmehr nur gleiches Recht für alle gelten lassen würde.

Für welche Strategie auch immer man sich in allen diesen Hinsichten entscheiden mag - in keinem Falle kommt man darum, sich auf das genaueste und gründlichste mit den einschlägigen Rechtstatsachen vertraut $\mathrm{zu}$ machen. Es folgt dies als erstes daraus, daß das Völkerrecht als praktisch sanktionsloses Recht die Spannung von Norm und Wirklichkeit geringer halten muß als jede andere Art von Rechtsordnung: Überspannung würde hier lediglich den Ungehorsam fördern,

1 So Wolfgang Graf Vitzthum, in Europa Archiv 1976, S. $129 \mathrm{ff}$. 
wenn nicht geradezu zu ihm nötigen². Im öffentlichen Seerecht kommt zweitens hinzu, daß man hier stärker als anderswo der Parole zu huldigen scheint „Ex factis jus oritur". So hat man das Recht des Anliegers auf einen Kontinentalschelf aus Geographie und Geologie gerechtfertigt, die den Sockel als Fortsetzung des Festlandes und daher "natürlich“ dem Anlieger zukommend zu erweisen hatten. In derselben Materie hat man die Ausdehnung dieses Rechtes geknüpft an die technische Möglichkeit, den Sockel von der Oberfläche her auszubeuten - der klassische Fall einer bedingungslosen Kapitulation des Rechts vor den jeweiligen Tatsachen. Eine Ordnung schließlich, die wie es bisher der Gemeingebrauch kraft ungeschriebenen Rechts regelte, an den bestimmungsgemäßen Nutzungen jedermann einen gleichen Anteil („Gemeinverträglichkeit“) sichern soll, muß vor allem darauf abheben, was an Nutzung insgesamt beansprucht wird und was angesichts des Übersteigens der Ansprüche gegenüber den Möglichkeiten der Befriedigung im ganzen und im einzelnen zugestanden werden kann. Als "Rechtstatsachen" sind hier vor allem also die Bestände an toten und lebendigen Schätzen in ihrer unablässigen Veränderung festzustellen und zu beurteilen, insbesondere insofern, als Raubbau ausgeschlossen werden muß.

Von allen hier angezeigten Schriften kann man rühmen, daß sie der Erforschung der Rechtstatsachen die gebotene Aufmerksamkeit geschenkt haben. Dieses Lob kommt vor allem Wolfgang Graf Vitzthum zu. Sein Werk über den „Rechtsstatus des Meeresbodens" beschäftigt sich beinahe zur Hälfte mit den bei einer Normierung zu berücksichtigenden Fakten: Es befaßt sich in einem ersten von zwei Teilen mit dem "Naturwissenschaftlichen und wirtschaftlichen Sachverhalt“. Er verrät hier eine beachtliche Sachkunde: Zuweilen hat man den Eindruck, es mit einer geologischen Darstellung zu tun $\mathrm{zu}$ haben, so daß man über die Rechtstatsachen vollständig ins Bild gesetzt ist. Dasselbe gilt zweitens von dem Bericht über die "Nutzung des Meeresbodens“ - ein Kapitel, das gewiß nur unter Überwindung beträchtlicher Informationsschwierigkeiten bewältigt werden konnte ${ }^{3}$. Hervorzuheben ist nicht zuletzt, daß auch der Möglichkeiten militärischer Nutzung des Meeresbodens gedacht wird.

In einer Zeit des Überganges vom „klassischen“ $\mathrm{zu}$ einem $\mathrm{zu}$ findenden neuen Offentlichen Seerecht kann keine einschlägige Darstellung umhin, sich nach der Darlegung der Rechtstatsachen Gedanken de lege ferenda $\mathrm{zu}$ machen und sie vorzutragen. Zwei der hier besprochenen Werke lassen sich diese Obliegenheit insbesondere angelegen sein. Es handelt sich einmal um das unter dem Stichwort „Rechtstatsachen“ bereits erwähnte Werk des Grafen Vitzthum: Die letzten fast 50 Seiten sind mit Vorschlägen zur Rechtspolitik erfüllt. Zweitens ist es Jochen Ehmer, der sich im Zusammenhang mit der Meeresverschmutzung um Überlegungen de lege ferenda bemüht: Abermals sind es fast 50 Seiten, die zum Abschluß hierauf verwendet werden. Beide Autoren mögen es dem Rezensenten nachsehen, wenn er hierauf lediglich ein für allemal verweist: In dieser Hinsicht kommt es zur Zeit mehr auf das an, was gelegentlich der Konferenz an Vorschlägen hervorgetreten ist.

2 Vgl. Herbert Krüger, Das Prinzip der Effektivität, oder: Uber die besondere Wirklichkeitsnähe des Völkerrechtes (1957), jetzt in: Staat - Wirtschaft - Völkergemeinschaft (Frankfurt am Main 1970), S. $121 \mathrm{ff}$. 
Man wird inzwischen vielleicht sogar etwas ungeduldig fragen: Wie steht es denn eigentlich mit der Freiheit der Meere? Wie geht es diesem Patienten? Hat er Aussicht, zu überleben, wenn auch hier nicht gerade in der alten Kraft und Fülle? In diesem Zusammenhang ist die unten vorgeführte Arbeit Jochen Ehmers $\mathrm{zu}$ nennen. Bevor sie auf ihr eigentliches Thema, die Meeresverschmutzung eingeht, prüft sie ungemein sorgfältig, ob die juristische Voraussetzung beliebiger Einbringung von Abfall, Müll usw. in das Meer, die Freiheit der Meere noch besteht. E. bezweifelt nicht, daß dieses Ideal unverändert hochgehalten wird. Zwar ist dieser Rechtsgrundsatz vielfach mißachtet und gefährdet. Aber der Grundsatz als solcher wird nach E. nicht bestritten.

Die Prognose, die man der Freiheit der Meere zu stellen hat, muß heute wesentlich ungünstiger ausfallen, als sie der Rezensent im Dezember 1959 noch stellen konnte ${ }^{4}$. Es wird sich dies vor allem aus der im folgenden vorgeführten Literatur über die neuen Erscheinungen "Wirtschaftszone“ und "Meeresbergbau“ ergeben. $\mathrm{Zu}$ fürchten hat man vor allem für die "Schiffahrtsfreiheit“, die die älteste, wichtigste und zugleich harmloseste Nutzung der Meere darstellt. Es ist daher begreiflich, daß man dieser "Freiheit" und ihrem Schicksal erhöhte Aufmerksamkeit widmet. Das aus dieser Sorge entsprungene Sammelwerk soll daher den Reigen der im folgenden vorgestellten Arbeiten zum Seevölkerrecht eröffnen ${ }^{5}$.

\section{Schiffahrtsfreiheit}

B.

Bernhardt Rudolf (Hrsg.). Die Schiffahrtsfreiheit im gegenwärtigen Völkerrecht Berichte einer Studientagung der Deutschen Gesellschaft für Völkerrecht (Karlsruhe 1975).

Unter Schiffahrtsfreiheit ist diejenige Nutzung der Meere zu versehen, die jedermann deren Befahren mit Schiffen aller Art, also auch mit Kriegsschiffen, und die dieserhalb erforderlichen Nebentätigkeit, wie z. B. Ankern, ohne irgendeine Erlaubnis gestattet, und zwar gleichgültig, ob es sich um das Küsten- oder das Hohe Meer handelt. Insofern diese Gestattung ein Ausfluß des Gemeingebrauches am Hohen Meer ist und dieser Gemeingebrauch einmal durch die Zweckbestimmung der Meere und zweitens durch die Gemeinverträglichkeit, d. h. das gleiche Recht aller zu gleicher Nutzung beschränkt ist, bietet die Freiheit der Schiffahrt die geringsten Schwierigkeiten: Schiffahrt ist die der Bestimmung der Meere gemäßeste Nutzung, und eine Problematik der Gemeinverträglichkeit ergibt sich heute weniger aus der gleichen wie aus andersartigen Nutzungen, z. B. der Ausbeutung des Kontinentalsockels. Hierin hat man wohl den vornehmsten Grund dafür zu sehen, daß die Schiffahrtsfreiheit im Rahmen der umfassenden Neuordnung des öffentlichen Seerechtes so gut wie unbestritten ist und daß ihre Gefährdung eine mittelbare ist, insofern es andere und neue Nutzungen sind, die sie praktisch in

3 Vgl. hierzu auch Harald Bäcker, Erzschlämme - Auf der Suche nach neuen Rohstoffquellen im Meer in: "Neue Zürcher Zeitung" vom 25. Mai 1976, S. 33.

4 Vgl. H. Krüger, Die Freiheit der Schiffahrt im Licht der Vereinheitlichung des Seerechts. Privatdruck der Deutschen Schiffahrtsbank A.G. Bremen (1960).

5 Eine gedrängte Darstellung der Fragen und Standpunkte, die das aktuelle öffentliche Seerecht betreffen, gibt Gerhard Breuer, Neue Entwicklungen des Seerechts - Schriften des Deutschen Vereins für Internationales Seerecht, Reihe A: Berichte und Vorträge Heft 22 (Hamburg 1975). 
Frage stellen. Das Schiff ist anspruchslos: Es nimmt nur einen kleinen Teil der Meeresoberfläche und auch diesen nur für ganz kurze Zeit in Anspruch. Die Ausbeutung des Meeresbodens hingegen benötigt große Felder, und die ihr gewidmeten festen oder beweglichen Anlagen besetzen große Flächen des Meeres. Diese Verschiedenheit verschärft sich zudem ungemein, wenn man - wie es bereits geschehen ist - die Gemeinverträglichkeit durch den Vorrang des Meeresbergbaues zu ersetzen sucht. Es mag dahin kommen, daß der Schiffahrt lediglich das verbleibt, was der Meeresbergbau übrigläßt - was nichts anderes heißen würde, als die natürliche Ordnung der Dinge auf den Kopf stellen.

Schon diese Möglichkeit allein rechtfertigte es, daß die Deutsche Gesellschaft für Völkerrecht im Herbst 1974 dem Thema „Die Schiffahrtsfreiheit im gegenwärtigen Völkerrecht" eine Studientagung gewidmet hat. Die hier vorgetragenen Referate liegen nunmehr in einem Sammelband vor. Vorweg gibt Walter Rudolf einen allgemeinen, unterrichtenden Überblick über „Die Freiheit der Handelsschiffahrt und ihre aktuellen Gefährdungen“. Diese Gefährdungen bilden den Gegenstand der folgenden Referate. Hieran soll die folgende Besprechung anknüpfen.

\section{Das Küstenmeer}

Hiermit befassen sich die von Dieter Rauschning ${ }^{6}$ und Ondolf Rojahn ${ }^{7}$ erstatteten Berichte. In diesem Zusammenhang handelt es sich einmal um die Ausdehnung dieses Streifens ins Hohe Meer hinein, mit der Folge einer entsprechenden Verkleinerung des letzteren. Dieser Vorgang ist einmal bedenklich, weil die Staaten durchweg ein Recht usurpieren, diese Ausdehnung einseitig festzusetzen. Diese Ausdehnung mag zugleich mit einer qualitativen Veränderung der Rechtslage verbunden sein, insofern zwar das Recht unschädlicher Durchfahrt und ebensolchen Überfliegens gewährleistet wird, der Modus von beidem aber ebenfalls der einseitigen Veränderbarkeit durch den Anliegerstaat unterworfen wird. Es ist daher wichtig, daß die Rechtswidrigkeit einer derartigen Einseitigkeit herausgestellt wird (Rauschning 41, 53) und daß nicht zuletzt die Erschleichung eines Küstenmeeres verurteilt wird, indem man die Ausdehnung nicht vom Großverlauf der Küstenlinie, sondern von womöglich deswegen errichteten künstlichen Inseln bemißt (Dicke a. a. O. 287, 293). Schließlich gehört hierher das von Inselstaaten in die Welt gesetzte Rechtsinstitut "Archipelagos", das gewaltige, vor allem für die Verbindung vom Indischen zum Pazifischen Ozean wichtigste Wasserstraßen einem einzelstaatlichen Regime unterwerfen würde, von dem nicht einmal sicher ist, ob es die „Unschädliche Durchfahrt" zugesteht ${ }^{8}$.

Werden Ausdehnung und Regime des Küstenmeeres einseitig vom Anlieger kraft seiner Souveränität festgestellt, dann ist die Gewährleistung „Unschädlicher Durchfahrt" für jedermann eine einseitige Vergünstigung, die eben als solche von seinem Standpunkt aus jederzeit und ohne Grund zurückgenommen oder geändert werden kann. Solange sie besteht, bietet diese Formel einen weiten Spielraum für Ausfüllung nach Gutdünken des Anliegers. Mag „Durchfahrt“ auch noch einigermaßen eindeutig sein, so läßt sich schwerlich etwas denken, was nicht als „schäd-

6 Die Durchfahrt durch nationale Gewässer vor den Küsten, S. 39-54.

7 Die Durchfahrt durch Internationale Meerengen, S. 79-110.

$\mathrm{S}$ Dieser Gegenstand wird in dem Sammelwerk mehr beiläufig behandelt; vgl. Rauschning 49, 52. 
lich" qualifiziert werden könnte. In diesem Zusammenhang hat man vor allem auf die Verantwortung des Anliegers gegenüber der Umweltverschmutzung hingewiesen. Diese Verantwortung soll eine weitgehende Pflicht zur Prävention einschließen, die etwa Konstruktion usw. von Tank-oder Sonderschiffen ${ }^{9}$ gelten würde.

Man sieht jetzt schon: Es ist die Einseitigkeit, die eine für die Schiffahrt unerträgliche Rechtsverschiedenheit und eine ebensolche Rechtsunsicherheit erzeugen würde. Eine Einigung qua Seerechtskonferenz hätte daher auf jeden Fall den Vorzug, wenigstens Rechtseinheit und damit Rechtssicherheit zu schaffen. Würde beides allerdings mit einer unfreiheitlichen oder freiheitsgefährdenden Regelung erkauft, dann wäre dieser Vorzug wieder zunichte gemacht. Vielleicht wirkt es sich hier zugunsten der Freiheit aus, daß die Neuen Nationen gerade auch schiffahrttreibende Nationen werden wollen und daß sie daher nicht wohl jetzt Fesseln schaffen können, die sie alsbald selbst behindern werden.

In diesem Rahmen schafft schon die Ausdehnung des Küstenmeeres auf 12 sm eine spezielle Problematik für die Meerengen: Nicht weniger als 121 ihrer Gattung würden durch eine solche Ausdehnung neu entstehen (Rojahn 85). Dies ist für die Handelsschiffahrt weniger von neuem Gewicht als für die Kriegsflotten der beiden Weltmächte. Sie wollen sich nicht mit dem Recht auf „Unschädliche Durchfahrt“ begnügen, obwohl Kriegsschiffe hiervon nicht grundsätzlich ausgeschlossen sind. Sie bestehen vielmehr auf „Freiem Transit“, d. h. der Beibehaltung des Rechtszustandes, wie er bestand, als diese Meerengen noch dem Hohen Meer zugehörten und wie er jede Reglementierung durch einen anderen als den Flaggenstaat ausschloß (Platzöder ${ }^{10}$ 118). Angesichts der Unvereinbarkeit beider Standpunkte kann diese Frage nur machtpolitisch entschieden werden (Platzöder 131). Hier scheint sich die. Waage keineswegs zuungunsten der Seemächte zu neigen: Gerade die Entwicklungsländer scheinen sich darüber klar zu sein, daß nichts zustande kommen wird, wenn sie diesen machtpolitischen Notwendigkeiten nicht Rechnung tragen.

\section{Festlandssockel11}

Zwar begründet dieses Institut keine Herrschaft über die über ihm ruhende Wassersäule, und damit scheint es eine Gefahr für die Schiffahrt nicht darstellen zu können. Abermals ist es jedoch die mittelbare Wirkung, die auch hier in Betracht zu ziehen ist. Dies wird gefordert einmal von den zur Ausbeutung der im Boden lagernden Schätze erforderlichen Anlagen: $\mathrm{Da}$ solche Schätze vor allem in diesem Teil des Meeresgrundes sich finden, sind solche Anlagen imstande, die Meeresoberfläche durchaus mit Verkehrshindernissen zu bestücken ${ }^{12}$. Da die Nutzung des Bodens durch Bergbau der Nutzung der Fläche durch Schiffahrt gleichrangig ist, entscheidet im Zweifel der Grundsatz der zeitlichen Priorität (Lagoni 273, Graf

\footnotetext{
9 Nach Norbert Peltzer, Aktuelle internationalrechtliche Probleme der friedlichen Reaktorschiffahrt a. a. O., S. $321 \mathrm{ff} ., 333$, ist ihre Durchfahrt eine "unschädliche“.

10 Renate Platzöder, Die Behandlung der Meerengenfrage auf der Dritten Seerechtskonferenz der Vereinten Nationen - Caracas Session 1974, S. $111 \mathrm{ff}$., 142.

11 Bernd Rüster, Die Ausbeutung des Festlandsockels und der ausschließlichen Wirtschaftszone in ihrer Bedeutung für die Schiffahrtsfreiheit, S. 233-264. Rainer Lagoni, Einrichtungen des maritimen Bergbaues und die Schiffahrtsfreiheit in Nord- und Ostsee, S. 263-184. Wolfgang Graf Vitzthum, Die- Ausbeutung des Meeresbodens in ihrer Bedeutung für die Schiffahrtsfreiheit, S. 303-320.

12 Bernd Rüster, S. 259, Anm. 70, hält es für denkbar, „daß man eines Tages nur noch mit Lotsen durch Schelfausbeutungsgebiete wird fahren können“.
} 
Vitzthum 314). Insofern das einzelne Schiff notwendig immer später kommt, wirkt sich diese Konfliktregelung ebenso notwendig zugunsten der Anlagen zur Ausbeutung des Meeresgrundes aus. Immerhin scheint hier im Einzelfalle ein Kompromiß in Gestalt von festen Schiffahrtswegen möglich ${ }^{13}$. Insgesamt kommt Lagoni (261) zu dem Ergebnis, daß der wachsende Umfang des marinen Bergbaues die Schiffahrt in immer größeren Seegebieten be- oder gar verhindern wird.

Als zweites kommt hinzu, wie vor allem Bernd Rüster hervorhebt, die Rechtsunsicherheit, unter der das geltende Recht leidet. Besteht sie für das Küstenmeer in der Undeutlichkeit des Begriffes „unschädlich“ und wird ihr nicht einmal durch eine Vermutung zugunsten der Unschädlichkeit (sc. der Durchfahrt) ein wenig abgeholfen, so ist es für den Festlandsockel als erstes die Ungewißheit darüber, wie weit hinein er in das freie Meer reicht: Es ist keine Glanzleistung der International Law Commission der Vereinten Nationen gewesen, wenn die Bestimmung der Ausdehnung zweitens in Abhängigkeit gesetzt wird von der technischen Möglichkeit der Ausbeutung von der Oberfläche her (Rüster 242) - im übrigen nichts anderes als eine bedingungslose Kapitulation des Rechtes vor der Technik, nichts anders, als wenn die Geschwindigkeit auf den Straßen danach bestimmt würde, was die Kraftwagen zu leisten vermögen. Die Unsicherheit über die Ausdehnung wird vermehrt durch die Ungewißheit über das innerhalb dieser Zone geltende Recht: Das Recht der Ausbeutung ist nämlich nicht allgemeines Völkerrecht, sondern jeweiliges Landesrecht ${ }^{14}$. Man darf schließlich nicht übersehen, daß nicht überall der Grundsatz der Gesetzmäßigkeit der Verwaltung gilt, so daß man auf unberechenbare Einzelmaßnahmen der Verwaltung gefaßt sein muß.

Als drittes ist zu bedenken, daß zwar der Rechtstitel „Festlandsockel“ lediglich ein Recht zur Ausbeutung der in demselben ruhenden Lagerstätten vermittelt, daß es aber gewiß nicht der erste, der Rechtsgeschichte bekannt werdende Fall wäre, wenn dieses Teilrecht sich zum Vollrecht entwickeln würde (Rüster, S. 262, 263). Diese Befürchtung hat sich zwar nicht in einzelnen Fällen, wohl aber institutionell erfüllt insofern, als man das Institut "Wirtschaftszone“ geschaffen und ihm in kürzester Zeit weitgehende Anerkennung verschafft hat. Da dieses letzte Institut den umfassenderen Titel darstellt, also die Ausbeutung des Sockels einschließt, kann man mit Rüster (257) vermuten, daß das Konzept „Festlandsockel“ in absehbarer Zeit nur noch völkerrechtsgeschichtliches Interesse wird beanspruchen dürfen. Sollte damit die vom Titel „Festlandsockel“ qua Merkmal „Ausbeutbarkeit“ ausgehende Gefahr eines Aufgehens des gesamten Meeresbodens und seiner Naturschätze in diesem Titel gebannt sein, so ließe sich dieser Entwicklung vielleicht eine positive Seite abgewinnen.

Hier scheint schließlich die rechte Stelle gegeben, der rechtlichen Problematik der Bohrinseln $\mathrm{zu}$ gedenken ${ }^{15}$. Sie führt aus dem Seerecht hinaus in die Allgemeine Staatslehre hinein. So wirft D. die Frage auf, ob eine große, ständig mit Menschen besetzte Bohrinsel als Staat angesprochen werden muß (291 f.) -, wenn auch einstweilen lediglich als Piratenstaat, solange nämlich, wie die Insel keine anerkannte

\footnotetext{
13 Lagoni 277, 281. 14 Bernd Rüster hat sich durch eine Edition der „Verträge und Deklarationen über den Festlandsockel (Continental Shelf) " verdient gemacht (Dokumente, herausgegeben vom Institut für Internationale Angelegenheiten Hamburg usw. Heft 42, Frankfurt am Main 1975). Eine Ubersicht über dieses Material gibt Rüster auch in seinem Beitrag $\mathrm{zu}$ dem hier angezeigten Sammelwerk, S. 243, Anm. 26. Wohl mit bemerkt Rüster (246), daß es dieser Gesetzgebung mehr um die Förderung der Ausbeutung der Sockelschätze als um die Freiheit der Schiffahrt gehen wird.

15 Detlev Chr. Dicke, Die staatsrechtliche Problematik von Bohrinseln, S. 285 ff.
} 
eigene Flagge führt (300). Wäre die Staatlichkeit als solche zu bejahen, dann würde sich die Frage nach der Legitimität dieses Staates stellen, inwiefern also die Gründer, insbesondere etwa ein multinationales Unternehmen, zu solcher Vaterschaft völkerrechtlich legitimiert sind $(285,287)$.

\section{Wirtschaftszone ${ }^{16}$}

Dieses Rechtsinstitut unterscheidet sich von dem Rechtstitel „Festlandsockel“ grundlegend dadurch, daß es alle wirtschaftlichen Nutzungen dem Anliegerstaat vorbehält, die in dieser Zone möglich sind und möglich sein werden, gleich ob es sich um die Wassersäule oder den Meeresgrund handelt.

Dieses Institut richtet sich damit vor allem gegen den Fernfischfang. Da sich ertragreiche Fischgründe überwiegend in dieser Zone finden, ist es zudem geeignet, diese Art von Fischerei auf das empfindlichste zu treffen. Damit allein ist jedoch der oft und gern gegen die Entwicklungsländer erhobene Vorwurf der wirtschaftspolitischen Freiheitsfeindschaft und einer fatalen Neigung für „Dirigismus“ nicht gerechtfertigt. Es sind vielmehr die modernen Fangmethoden und ihre Fähigkeit, ganze Fischpopulationen, wie z. B. den Hering in der Nordsee, praktisch auszurotten, die schon seit langem eine Kultur der Freiheit fordern und zu Abkommen geführt haben, deren Einhaltung allerdings, wie z. B. im Falle der Wale, nicht eben als eine strikte bezeichnet werden kann. Angesichts der Notwendigkeit, der Überfischung entgegenzutreten, bleibt nur die Frage, ob dies räumlich, also universal, regional oder national erfolgen soll, oder ob man sich an dem Schutzgut, also einzelnen von Ausrottung bedrohten Fischarten, wie z. B. den Thunfisch, $\mathrm{zu}$ orientieren hat. Mit der Entscheidung für die Wirtschaftszone entscheidet man sich für eine nationale Lösung dieses internationalen Problems. Es ist nicht auszuschließen, daß man damit vom Regen in die Traufe kommt, zumal wenn die Hege und Pflege der Meeresfische vor allem wegen ihrer Wanderungen national mit durchschlagendem Erfolg nicht betrieben werden könnte.

Was die Schiffahrtsfreiheit (und die Freiheit des Überfliegens) angeht, so beteuern alle Verfechter der Wirtschaftszone, daß das Recht der Anlieger durch die Pflicht zur Duldung von „Unschädlicher Durchfahrt" belastet sei. Die bereits für das Küstenmeer herausgearbeitete prekäre Natur dieser Freiheit dehnt sich damit von 12 auf 200 Seemeilen aus. Darüber hinaus muß gefragt werden, ob die Wirtschaftszone nicht auch eine qualitative Veränderung dieser Freiheit bewirken wird. In diesem Zusammenhang muß man sich darüber klar sein, daß mit der Wirtschaftszone insbesondere die Bekämpfung der Meeresverschmutzung weithin in die Hände der einzelnen, nunmehr womöglich qua Gebietshoheit zuständigen Nationen gelegt und damit dem Flaggenstaat weithin entwunden wird, womit die Schiffe insoweit unter ein Doppelregime gestellt sind ${ }^{17}$.

16 Günther Jaenicke, Die Fischereifreiheit auf der Hohen See, Entwicklungen bis zur 3. Seerechtskonferenz in Caracas, S. 163-194. Gerhard Hafner, Die Gefährdung der Freiheit der Hochseefischerei; das Urteil im isländischen Fischereistreit im Lichte der 3. Seerechtskonferenz der Vereinten Nationen, S. $195 \mathrm{ff}$. -232 .

17 Vgl. Rüdiger Wolfrum, Die Beschränkungen für die Freiheit der Schiffahrt durch das kanadische "Artic Waters Pollution Prevention Act" sowie die internationalen Ưbereinkommen zum Schutze der Meeresumwelt, S. $145-162$. 
Dies genügt bereits, um die Frage aufwerfen zu können, wodurch sich die Wirtschaftszone noch vom Küstenmeer unterscheiden würde. Zur Zeit streitet man sich darüber, ob sie trotz aller Rechte des Anliegers Teil der Hohen See bleiben würde oder ob sie als Gebilde sui generis zu konstituieren wäre. Im ersten Falle würde die Freiheit der Meere als die Regel, die Rechte der Anlieger als die Ausnahme erscheinen - mit der Folge, daß diese Rechte eben als Ausnahmen eng auszulegen wären - daß insbesondere, wie der Titel Wirtschaftszone es verlangt, diese Rechte sich auf die wirtschaftliche Nutzung zu beschränken hätten. Was allerdings als nichtwirtschaftliche Nutzung übrigbleibt, insbesondere wenn sich die Küstenstaaten auch die wissenschaftliche Forschung vorbehalten wollen, könnte nicht allzu erheblich ausfallen.

\section{Der Meeresboden ${ }^{18}$}

Es wird unterstellt, daß letztlich die Wirtschaftszone ein aus der "Ausbeutbarkeit“ abzuleitendes Recht der ökonomisch und technisch fortgeschrittensten Anlieger ${ }^{19}$ ausschließen wird, den Meeresboden unter sich aufzuteilen. Dies vorausgesetzt stehen für dessen Status zwei Möglichkeiten zur Diskussion: Entweder das Recht freier Okkupation für jedermann, also nicht etwa nur für Staaten; oder eine Internationalisierung mindestens so weit, daß eine Organisation der Völkerfamilie die Felder absteckt und vergibt. Auch hier wieder sind es die für die Ausbeutung von der Oberfläche her erforderlichen Anlagen, von denen die Schiffahrt für ihre Freiheit sicher de facto und vielleicht auch de jure zu fürchten hat. Wenn man die Schätze mit einer Art von Staubsauger aus großer Tiefe hervorholen wird, dann ist diese Technik gegenüber äußeren Einwirkungen, wie die Schiffahrt sie mit sich bringt, gewiß anfälliger als ortsfeste Anlagen. Es mag sein, daß man dieserhalb einen Vorrang des Meeresbergbaues vor der Schiffahrt begründen wird (hierüber Graf Vitzthum, 306). Selbst bei Gleichrangigkeit (vgl. Lagoni, 272) genießt aber der Bergbau die zeitliche Priorität: Was er vom Meer in Anspruch genommen und innehat, bleibt kraft Priorität allen anderen Nutzern damit verschlossen, und zwar unter Umständen für Jahrzehnte.

Einstweilen scheinen solche Gefahren jedoch nicht aktuell: „Noch ist die Ausbeutung des küstenfernen Unterwassergebietes keine Realität" (Graf Vitzthum, 303); überhaupt: „Die von der Ausbeutung des Meeresbodens drohenden Gefahren dürfen nicht dramatisiert werden" (derselbe 319; vgl. auch 312, 313).

\section{Meeresverschmutzung ${ }^{20}$}

Die von diesem Sachverhalt für die Schiffahrt ausgehenden Gefahren liegen weniger in ihm selbst als in seiner Bekämpfung. Wie unsere Flüsse zeigen, kann man auch auf Kloaken Schiffe halten und bewegen. Eine Ausnahme für das Meer macht allein die radioaktive Verseuchung durch Ablagerung von Atommüll oder durch Un-

18 Graf Vitzthum, Die Ausbeutung des Meeresbodens in ihrer Bedeutung für die Schiffahrtsfreiheit, S. $303 \mathrm{ff} .-320$. Vgl. auch denselben, Der Rechtsstatus des Meeresbodens (Berlin 1972) und die unten folgende Besprechung dieses Werkes.

$19 \mathrm{Im}$ übrigen muß man mit der Auffassung rechnen, daß die Spitzenleistung eines Anliegers allen zuzurechnen 1st.

$20 \mathrm{Vgl}$. Rüdiger Wolfrum und Ondolf Rojahn a. a. O. Hierzu auch unten die Besprechung von Jochen Ehmer, Der Grundsatz der Freiheit der Meere und das Verbot der Meeresverschmutzung. Dort auch weitere Literatur zu diesem Thema. 
fälle von spaltbares Material befördernden Schiffen oder Reaktorschiffen. Solche Gefährdungen mögen sich mittelbar zu einer Beeinträchtigung der Schiffahrtsfreiheit auswachsen, wenn sie ein Staat zum Anlaß von vorbeugenden Maßnahmen nimmt und dieserhalb seine Hoheit weit über das Küstenmeer in das Hohe Meer hinein ausdehnt. Dies hat - glücklicherweise bisher als einziger Staat - Kanada im Jahre 1970 getan. Wie für das Küstenmeer, so hängt auch für diese Art von Zonen alles davon ab, was der Anlieger zur Bekämpfung der von ihm ins Auge gefaßten Gefahren nach seinen Auffassungen und Maßstäben für erforderlich hält. Wolfrum urteilt hierüber: „Die praktische Bedeutung des kanadischen Gesetzes sollte nicht unterschätzt werden, denn es schränkt die Freiheit der Schiffahrt in nicht unbeträchtlichem Umfang ein“ (146). Und später heißt es: „Insgesamt läßt sich unter Berücksichtigung der geographischen Verhältnisse in dem zu den arktischen Gewässern gehörenden Meeresgebiet sagen, daß es mit Hilfe des kanadischen Gesetzes unschwer möglich ist, diese Region für die Schiffahrt generell zu sperren oder das Befahren doch zumindest sehr wesentlich zu erschweren" (147). Insofern die Herren von "Wirtschaftszonen“ sich die Verhütung von Meeresverschmutzung nicht minder angelegen sein lassen werden als die Kanadier, können diese Feststellungen allgemeine Geltung, und zwar nicht zu vergessen auch für das Küstenmeer beanspruchen.

Der Sammelband, dessen Besprechung hiermit abgeschlossen sein soll, zeigt, welch erfreulich hohe Zahl junger Wissenschaftler der BRD sich mit Offentlichem Seerecht beschäftigt. Die Darstellung ist durchweg gründlich, das Urteil wohlabgewogen. So kann man sagen, daß dieser Band einen wertvollen Beitrag für die Erneuerung des Seerechtes mit Vorzeichen einer auch in ihm zu erhaltenden und zu fördernden Freiheit der Schiffahrt darstellt.

C.

Wir wenden uns nunmehr der Würdigung von Einzelwerken zu, die die in dem besprochenen Sammelband behandelten Gegenstände monographisch bearbeitet haben.

\section{Wirtschaftszone}

Der Gedanke einer "Wirtschaftszone“ ist so jung, daß man es wohl hierauf zurückzuführen hat, daß eine ausführliche und gründliche Behandlung dieses Themas noch nicht vorliegt. Dies ist um so bedauerlicher, als sie trotz ihrer Jugend so weite Anerkennung, und zwar vor allem auch durch die zwei Weltmächte gefunden hat, daß man ihr alle Aussichten für eine Kodifizierung einräumen muß. Diejenigen Länder, denen dieses Institut wichtige Rechte, vor allem die Möglichkeit der Fischerei nehmen wird, haben sich dennoch zu einer solchen Haltung wohl durch die Erwägung bestimmen lassen, daß nur hierdurch sich retten läßt, was noch $\mathrm{zu}$ retten ist ${ }^{21}$. Hier kommt es zuerst darauf an $\mathrm{zu}$ verhindern, daß die Wirtschaftszone sich in der Sache zu einem zweiten Küstenmeer entwickelt, das

21 Vgl. Renate Platzöder, Zur Neuordnung des Meeresvölkerrechts auf der Dritten Seerechtskonferenz der Vereinten Nationen (Ebenhausen 1974), S. 6: „Je eher die grundsätzliche Anerkennung der Forderung nach Wirtschaftszonen erfolgt, desto erfolgreicher dürften die Anstrengungen um eine rechtliche Ausgestaltung dieser Zone sein.* 
sich von dem klassischen Küstenmeer nur durch einen anderen Namen unterscheidet. Auf die Verhinderung eines solchen Zusammenfließens zielen diejenigen, bereits erwähnten Bestrebungen, die die dem Herren einer Wirtschaftszone zustehenden Rechte aufzählen wollen - mit der zweifachen Folge, daß die Vermutung für die Freiheit der Wirtschaftszone spricht und zudem die Nutzungen als Ausnahmen eng zu verstehen sind: Beides wäre in der Tat das Gegenteil des dem Herren des Küstenmeeres eingeräumten Rechtsstatus, insofern Herrschaft hier die Regel und Beschränkung durch „Unschädliche Durchfahrt“ die Ausnahme ist. Gerade hierfür wäre es wichtig die Gründe genau zu kennen (außer dem „stat pro ratione voluntas"), die die Forderung nach einer Wirtschaftszone tragen, und ebenso die Vorstellungen, die über die Rechtsordnung einer solchen Zone umgehen. Nicht übersehen werden dürfen gerade auch in diesem Zusammenhang die nicht weniger als 52 Staaten, die nicht über eigene Küsten verfügen und die sich daher nicht nur wie schon 1958 ein Recht auf Zugang zum Meer, sondern vor allem auch einen Anteil bei der Aufteilung der Nutzungen des Meeres zu sichern wünschen $^{22}$.

Als einer der Vorläufer der Wirtschaftszone muß das Recht angesehen werden, das sich vor allem die sog. CEP-(Chile, Ecuador, Peru)-Staaten selbst als Reaktion auf die sog. den Festlandsockel erklärende Truman-Doktrin verliehen haben - das Recht auf eine sich $200 \mathrm{sm}$ in die Hohe See hinein erstreckende Zone, die den Anliegern zunächst einmal die Fischerei vorbehalten sollte. Hierüber unterrichtet das Ehepaar Smetherman ${ }^{23}$. Die Ursache dieses Ausgriffes sehen die Verfasser zunächst in der Befürchtung der Anlieger, die Industriestaaten könnten die für diese Länder lebenswichtigen Fischbestände dezimieren oder gar ausrotten. Anlaß zu solcher Befürchtung hat zuerst die Tatsache gegeben, daß vor diesen Küsten Jahr für Jahr 15000 bis 20000 Wale von Dritten erlegt worden sind $(14,21,38)$. Dieser Anlaß erklärt auch, warum man sich für eine Ausdehnung von gerade $200 \mathrm{sm}$ entschieden hat - eine Ziffer, die auch für die Wirtschaftszone maßgebend sein soll: Es ist dies die Breite der Humboldt-Strömung, deren Plankton den Anchovis Nahrung gibt, von denen 10 Mill. Tonnen zu Fischmehl verarbeitet werden, das vornehmlich in den Export geht. $\mathrm{Zu}$ solchen wirtschaftlichen Motiven kommen nationale Empfindlichkeiten: Es hat diese Entwicklungsländer verdrossen, daß sich entwickelte Länder, vor allem USA dank hochmoderner Fangtechniken, vor ihren Küsten so provozierend erfolgreich betätigen konnten $^{24}$. Dieses Feuer ist noch weiter angefacht worden durch die USA-Politik, auf die Ausdehnung der Herrschaft der CEP-Staaten mit Retorsionen zu reagieren, etwa mit der Versagung von Entwicklungs- und Militärhilfe (sehr aufschlußreich geschildert, S. $100 \mathrm{ff}$.). Es gelang den CEP-Staaten, in diesem Zusammenhang der USA vor der Organisation der Amerikanischen Staaten eine moralische Niederlage beizubringen (Verurteilung wegen Aggression). Unsere Verfasser weisen mit Recht darauf hin, daß auf Seite der CEP-Staaten das Bedürfnis nach Selbstdarstellung und Selbsterhebung entscheidend mitgewirkt hat: Letztlich ging es

22 Vgl. Kontroversen und Kompromisse um das Seerecht, in: „Neue Zürcher Zeitung “, vom 19. Mai 1976, S. 5

23 Territorial Seas ans Inter-American Relations - with Case Studies of the Peruvian and U. S. Fishery Industries (New York usw. 1974). Vgl. auch Ondolf Rojahn, Die Ansprüche der lateinamezikanischen Staaten auf Fischerei-Rechte jenseits der zwölf-Meilen-Grenze (1972).

24 In solcher Sicht mag die Freiheit der Meere geradezu als eine Art von Meeres-Kolonialismus erscheinen (S. 1). 
nicht mehr um Fische, sondern um die Souveränität (für Peru, S. 42 ff.; für Ecuador, S. 46 ff.). Bei dieser Gelegenheit tritt auch ein etwas kurioser Topos zutage: Ist es dem Einzelstaat eines Bundesstaates von Staats- und Völkerrechts wegen gestattet, seinerseits und für sich Herrschaft am Hohen Meer zu begründen, wie es USA-Einzelstaaten unternommen haben? (111). Es versteht sich von selbst, daß eine solche Möglichkeit unter beiden Aspekten zu verneinen ist. Inzwischen ist auch dieses Problem, jedenfalls für die USA, dadurch gegenstandslos geworden, daß diese Großmacht sich eine Fischereizone von 200 sm selbst verliehen hat ${ }^{25}$.

\section{Der Meeresboden und dessen Nutzung}

$\mathrm{Zu}$ diesem Gegenstand liegen zwei Monographien ${ }^{26}$ vor, die im folgenden besprochen werden sollen.

I. Von der ausführlichen und gründlichen Darlegung der Rechtstatsachen, die die erste Arbeit auszeichnet, ist bereits im allgemeinen Teil dieser Sammelbesprechung die Rede gewesen. Die im engeren Sinne juristische Hälfte (S. 156 ff.: Der Rechtsstatus des Meeresbodens) ist in der Hauptsache in drei Abschnitte gegliedert.

Der erste von ihnen berichtet über die "Gebietsrechtliche lex lata" (174 ff.). Hier geht es im Grunde allein um eine einzige Frage: Gibt es den Meeresboden als rechtliche Erscheinung überhaupt noch? Oder jedenfalls: Wird ihm nicht in absehbarer Zeit die Ausdehnung des Festlandssockels in das Hohe Meer hinaus den Garaus machen? Ausgelöst werden diese Fragen durch die zweite Bestimmung, von der die Konvention von 1958 die seewärtige Ausdehnung abhängen läßt: Wenn hiernach unter diesem Titel die Herrschaft über das Hohe Meer so weit ausgedehnt werden darf, wie die Ausbeutung des Sockels von der Oberfläche her technisch möglich ist - wenn andererseits die technische Entwicklung die Möglichkeit einer solchen Ausbeutung selbst bis in die größten Tiefen demnächst möglich gemacht haben wird - dann sind allerdings die Tage eines besonderen Meeresbodens und erst recht die Möglichkeit von dessen freier Ausbeutung gezählt. Angesichts solcher Aussichten hält es der Verfasser mit Recht für geboten, unter allen nur denkbaren Gesichtspunkten und so gründlich den Beweis dafür zu führen, daß selbst unter diesem Titel die Herrschaft nicht beliebig weit in das Hohe Meer und seine Böden hinaus ausgedehnt werden darf, daß vielmehr diese Ausdehnung auf eine Grenze stößt, jenseits deren das besondere und eigenartige Gebilde „Meeresboden“ mit einem ebenso besonderen und eigenen Recht beginnt - eine Grenze, von der man allerdings einstweilen nicht mehr sagen kann, als daß sie zwischen der Linie, die durch eine Wassersäule von $200 \mathrm{~m}$ bestimmt wird, einerseits und dem Fuß des Kontinentalsockels andererseits liegt (225). Graf Vitzthum selbst scheint einer festen Ziffer von 40 sm zuzuneigen, weil diese die durchschnittliche Ausdehnung des Schelfs bezeichnet (222).

25 Vgl. „Neue Zürcher Zeitung ${ }^{\star}$, vom 21./22. 3. 1976: „Das Gesetz besagt im wesentlichen, daß ausländische Fischer innerhalb der neuen 200-Meilen-Grenze nur mit Genehmigung der amerikanischen Behörden oder gemäß bilateral ausgehandelten Verträgen fischen dürfen. ${ }^{\alpha}$

26 Wolfgang Graf Vitzthum, Der Rechtsstatus des Meeresbodens (Berlin 1972). Evan Luard, The Control of the Sea-Bed - A new international issue - (London 1974). 
Man muß den vom Grafen Vitzthum für die juristische Existenz eines besonderen Meeresbodens angetretenen Beweis als voll geführt ansehen. Folgerichtig wird der Rechtsstatus dieses Meeresbodens bestimmt als der eines „zwingend hoheitsfreien Gebietes“ (233 ff.). Hieraus wiederum ergibt sich ein Okkupations- und Aneignungsverbot als bereits geltendes Völkerrecht (243). „Der Meeresboden besitzt in der Gebietslehre des Völkerrechts einen Sonderstatus" (vgl. das "Ergebnis“ S. 247).

Hieran schließt sich zweitens die Behandlung der lex lata, soweit es sich um die Nutzung dieses Meeresbodens handelt. Eine Erörterung dieses Komplexes ist durch die Ergebnisse nicht gegenstandslos geworden, die die Erforschung des Rechtsstatus des Meeresbodens gezeitigt hat: Denn aus diesen Ergebnissen wird man folgern müssen, daß die Nutzung des Meeresbodens wie die der Hohen See durch das Institut „Gemeingebrauch"27, und zwar auch hier nach dessen qualitativer wie quantitativer Seite, geregelt ist. Graf V. schlägt einen anderen Weg ein, um $\mathrm{zu}$ erkunden, ob es eine geltende Regelung der Nutzung des Meeresbodens gibt. Er stellt fest, daß das Ideal des „common heritage of mankind“ geltendes Völkergewohnheitsrecht geworden ist (268). Obwohl hiermit nach dem „Gemeingebrauch“ ein zweites, und zwar schwereres Hindernis für die „freie Ausbeutung des Meeresbodens aufgerichtet worden ist, zieht der Verf. hieraus genau den entgegengesetzten Schluß (263): Jeder "Erbe" darf das in Gesamthand stehende Gemeingut individuell ohne Verständigung mit oder auch nur Rücksicht auf die „Miterben“ nach Belieben nutzen und alle anderen, einschließlich seiner Miterben, von solcher Nutzung ausschließen (vgl. auch S. 293). Dem Verf. scheint zu entgehen, daß die Idee des Familiengutes der Menschheit damit auf den Kopf gestellt wird: In dieser Familie herrscht das brutale Recht des Stärkeren (vgl. S. 270: „Gewinnungsmonopol der Stärkeren") - bezeichnend hierfür ist, daß sowohl die USA wie USSR gegen eine Ausbeutungssperre gestimmt haben, wie sie dem Ideal eines Familiengutes vor einvernehmlicher Regelung der Nutzung entspräche, ja zwingend aus ihm folgte - und an die kommenden Generationen wird erst recht nicht gedacht, obwohl dies nicht zuletzt von dem Ideal „Familie“ gefordert wäre. Es nimmt hiernach nicht Wunder, daß Graf V. die Rechtsproblematik der Nutzung des Meeresbodens mit „Meeresbergbaufreiheit“ oder „Meeresbodenfreiheit" beantwortet.

Damit stellt sich sowohl de lege lata wie de lege ferenda die Frage: Setzt sich nicht eine Rechtsordnung mit sich selbst in Widerspruch, wenn sie auf die Regelung einer regelungsbedürftigen Materie verzichtet und sie dem „Recht des Stärkeren“ überantwortet? Oder ist sie hierzu allenfalls unter der Voraussetzung fähig, daß der Stärkere keine Schwächeren vorfindet, die er schädigen könnte, oder daß jedenfalls dem Stärkeren ein anderer Stärkerer entgegengesetzt wird, so daß beider Übermacht sich neutralisiert? „Freiheit des Meeresbergbaues" weiß von beiden Qualifikationen nichts: Sie institutiert schlicht und schlechthin das „Recht des Stärkeren“, das naturnotwendig zugleich das „Recht des Schnelleren“ ist (Wer zuerst kommt, mahlt zuerst! Vgl. S. 276 ff.). „Was die Schaffung einer internationalen Ordnung, betreffend den Meeresboden angeht, wünschen die Amerikaner die größtmögliche Freiheit der Forschung und Ausbeutung der Meeresschätze.

27 Wenn man mit Graf V. (280) den Meeresbergbau mit seinen ortsfesten Anlagen als "gesteigerte Sondernutzung " begreift, dann folgt hieraus, daß er insoweit nicht mehr unte
Gemeingebrauch fällt, daß es hierfür vielmehr eines besonderen Titels bedarf. 
Amerikanische Gesellschaften haben bereits Millionen von Dollars in diesem Bereich investiert. Die Entwicklungsländer indessen, die weder über die notwendigen Finanzmittel noch über die erforderliche Technologie verfügen, wenden sich gegen die von den Vereinigten Staaten angemeldeten Ansprüche . ..29". Zweifellos sind die Aussichten, daß eine internationale Behörde die Ausbeutung in die Hand nehmen wird oder jedenfalls allein Erlaubnisse erteilen kann, höchst unerfreulich: Die Annahme, daß sich hier Rechtsfragen nicht in Machtfragen verwandeln würden, erscheint illusionär. Unerfreulich ist aber auch die Aussicht auf ein neues „Wildwest", nicht zuletzt auch für die Investoren selbst: Wer sicher sie gegen Angriffe, vor allem auch im Namen des Rechts? Und würden in einem Seekrieg solche Anlagen nicht geprist werden dürfen? Am besten schiene es unter diesen und anderen Umständen (Meeresverschmutzung!), wenn auf die Ausbeutung des Meeresbodens allseitig verzichtet würde. Dem steht aber die Pleonexie, die "auri sacra fames“ entgegen. Allen diesen Mißlichkeiten will Graf V. durch materielle Normen und deren institutionelle Verwirklichung begegnen: Doch ist das einschlägige Nutzungsrecht so „offen" und vieldeutig, daß dem dringendsten internationalen Regelungs-, ja Verwaltungsbedürfnis de lege ferenda nur durch detaillierte Gesamtregelungen und organisatorische Neuschöpfungen genügt werden kann (309).

II. Das Werk Evan Luards ${ }^{29}$ ist in jeder Hinsicht von ganz anderer Art als das des Grafen Vitzthum. Als erstes beschränkt es seine Thematik nicht auf den Meeresboden, sondern dehnt diese auf Verhütung von Meeresverschmutzung und Erhaltung der Fischbestände aus - offenbar aus der Überzeugung heraus, daß der Meeresbergbau diejenige Nutzung ist, von der am stärksten eine Schädigung der Reinheit des Wassers und der Bestände an Fischen zu befürchten steht. Zu einer noch weiteren Ausdehnung der Thematik kommt es gelegentlich der Schilderung der Geschichte der internationalen Behandlung der ursprünglichen Gegenstände: Nunmehr werden alle noch übrigen Probleme des Seerechtes angesprochen und gewürdigt (128 ff.). Im Ergebnis ist es daher nicht allein der Meeresboden, sondern die gesamte Tagesordnung der Seerechtskonferenz, was den Gegenstand dieser Untersuchung ausmacht.

Auch L. beginnt mit den Rechtstatsachen. Diese Schilderung setzt ein mit einem Hymnus auf die Reichtümer, die der Meeresboden in sich birgt (Vorwort; S. 3 ff.; $6 \mathrm{ff} . ; 11 \mathrm{ff}$.$) . Besonders ärgerlich ist ihm daher die Gefahr einer Militarisierung$ des Meeresbodens: Was er hierüber zu berichten weiß, ist in der Tat alles andere als erbaulich (49 ff.). Es ist daher begreiflich, daß er diesem Gegenstand ein eigenes Kapitel 6 „The Disarmement Debate“ widmet (97 ff.). Er hält es daher für das wichtigste, daß die UN-Resolution von 1970 zugunsten ausschließlich friedlicher Nutzung des Meeresbodens überhaupt zustande gekommen ist (137). Der unter derselben Devise geschlossene, vor allem auch von den beiden Supermächten unterzeichnete Vertrag (108) erfüllt allerdings die Hoffnungen des Verfassers nicht: Dieser Vertrag, in Wirklichkeit weit entfernt davon, den Meeresboden ausschließlich einer friedlichen Nutzung vorzubehalten, schützt ihn nicht einmal vor Mißbrauch zu Zwecken der Kriegführung mittels Atomwaffen: „The fact is that

$28 \mathrm{Nach}$ „Neue Zürcher Zeitung ${ }^{\alpha}$ vom 22. Mai 1976.

29 Dieser Autor ist früher durch Arbeiten zur Außenpolitik bekannt geworden; vgl. z. B. Evan Luard (ed.) The Cold War - A Reappraisal - (New York 1964). 
the treaty does not even prohibit all uses of nuclear weapons fired from submarines or other vessels resting on the sea-bed: at an early stage after the introduction of the US-Soviet text on October 7, 1969, the U. S. delegate made unmistakably clear, as the treaty was concerned with the uses of the sea-bed and not of vessels on the sea-bed, a submarine anchored to, or resting on the sea-bed would not be violating the treaty" $\left(110 \mathrm{f} .{ }^{\mathbf{3 0}}\right)$.

Mit alledem ist vorweggenommen die Beantwortung der Frage, bei deren Verneinung alle anderen Probleme sich als gegenstandslos erweisen würden: Kennt das Völkerrecht den Meeresboden als ein unbedingtes und dauerndes Institut, oder existiert es lediglich prekär, so lange nämlich, bis die Küstenstaaten durch Ausdehnung des Festlandssockels unter dem Titel der „Ausbeutbarkeit“ den Meeresboden in eben diesem Sockel haben auf- oder untergehen lassen? L. verneint, daß besagter Titel eine beliebig weite Ausdehnung des Shelfs gestattet (41, 47, 48; vgl. auch $143 \mathrm{f}$. und $152 \mathrm{ff}$.) - ja er behauptet: „In many ways, therefore, the Convention (sc über den Festlandsockel) can become a valueless document." Im weiteren Verlauf der Darstellung glaubt man jedoch bemerken zu können, daß der Verfasser an dieser seiner These selbst zu zweifeln beginnt: Gewißheit für deren Richtigkeit, die er schon auf S. 41 bejaht hatte, scheint er letztlich erst aus der UN „Declaration of Principles Governing the Sea-Bed, and the Ocean Floor, and the Subsoil Thereof, Beyond the Limits of National Iurisdiction" (vom 17. Dezember 1970 Text S. $139 \mathrm{ff}$.) zu schöpfen. In der Tat schiene bereits die Überschrift dieser Deklaration sinnlos, wenn es einen „Meeresboden“ entweder überhaupt nicht gäbe oder jedenfalls sein nahes Ende mit Sicherheit vorauszusehen wäre (136, 139). Auch glaubt L. feststellen zu können, daß dies auch die unter der Mehrheit der Staaten herrschende Auffassung sei.

Der Rechtsstatus des Meeresbodens gemäß der lex lata erscheint dem Verf. noch unklarer als der des Festlandsockels. Vor allem: Wem gehört er? (34 ff., $127 \mathrm{ff}$.). Die Antwort findet sich S. 45: Keine Nation kann Anspruch auf den Meeresboden erheben. Die Folgerung, daß er jedermann zur Ausbeutung offenstehe, und zwar nach der Regel „first come first served“, scheint L. nicht hinnehmen zu wollen (129). Dieser Eindruck wird dadurch verstärkt, daß der Verf. der Idee des "Familiengutes der Menschheit" anhängt $(131,137)^{31}$, daher die Resolution über eine Sperre der Ausbeutung billigt und hiernach einseitige Ausbeutung schwerlich noch für zulässig hält (136).

Nicht der geringste Wert der Arbeit liegt darin, daß sie eine Vorgeschichte der Dritten Seerechtskonferenz bietet ${ }^{32}$. Das gilt insbesondere von der Darlegung der Interessen, der Standpunkte, der Vorschläge usw., die hier hervorgetreten sind, und zwar zu allen Punkten der Tagesordnung. Im Mittelpunkt steht auch hier das Thema „Meeresboden“ und hier wieder die Frage, ob er und seine Ausbeutung einem Internationalen Regime unterstellt werden sollen (169 ff.). Hierzu entwirft der Verf. als erstes ein Schema aller nur denkbaren Lösungen des Ausbeutungsproblems (170 ff.: notification, registration, allocation, international enterprise) und beurteilt hiernach die Vorschläge, die zu diesem Gegenstand in der zustän-

\footnotetext{
30 Ebenso Graf Vitzthum a. a. O., S. $122 \mathrm{ff}$.

31 Seltsamerweise hält der Verf. den USA-Präsidenten Johnson für den Vater dieses Gedankens - Malta soll ihn von Johnson übernommen haben! $(84,86,91)$

32 Vgl. unten die Besprechung des hierüber von Renate Platzöder verfaßten, bereits oben Anm. 21 erwähnten Werkes.
} 
digen UN-Kommission eingebracht worden sind (176 ff.): Während der Osten sich als ein entschiedener Gegner einer internationalen Behörde vor allem supranationalen Charakters erweist, widmet der Westen dieser Idee immerhin Lippendienste, während die Entwicklungsländer sie energisch bejahen. Insgesamt ergeben diese Materialien, daß man sich vor Beginn der Konferenz zu Klarheit allenfalls über die großen Umrisse durchgerungen hat, jedoch nicht zu einer Übereinstimmung gelangt ist: Der Verf. stellt nicht weniger als 25 „more important points of disagreement" zusammen(191). Sollte es zu einer Internationalisierung des Meeresbodens kommen, dann wird für Auslegung und Anwendung der einschlägigen Normen, die von L. festgehaltene Entstehungsgeschichte von Nutzen sein.

\section{Meeresverschmutzung ${ }^{33}$}

Der Verfasser geht nicht sogleich medias in res - er prüft vielmehr ausführlich (28 - 82), ob es die Freiheit der Meere de facto und de jure noch gibt. Würde es nur noch Küstenmeer geben, dann würden diejenigen Bindungen entfallen, die das allgemeine Seerecht gerade auch bezüglich der Verschmutzung kennt - jeder Anlieger würde seinen Meeresteil nach Gutdünken verschmutzen dürfen, und zwar ohne Rücksicht darauf, daß die Wirkungen hiervon sich nicht auf seinen Anteil beschränken: Ein völkerrechtliches Nachbarrecht zur See scheint es noch weniger zu geben als zu Lande. In dieser - für ihn - Vorfrage kommt der Verf. zu dem, allerdings nicht allzu ausdrücklich hervorgehobenen Ergebnis (82), daß - wenn auch stark verkürzt - die Freiheit der Meere als Grundsatz noch in Geltung stehe.

Hiernach ist das Problem dahin zu formulieren, ob und ggf. unter welchen Modalitäten die Freiheit der Meere das Recht auf Verschmutzung einschließt - wobei es sich allerdings von vornherein als mißlich erweist, daß „Verschmutzung“ nicht definiert, ja wohl nicht einmal definierbar ist (88). Hierbei kommt es nicht so sehr auf regionale Abkommen (Ostsee, Nordsee, Mittelmeer) als vielmehr darauf an, was das allgemeine ungeschriebene Seevölkerrecht als Regelung aufweist. Der Verf. geht davon aus, daß die freie Nutzung des Hohen Meeres sich auf das beschränkt, was das Institut "Gemeingebrauch“ gestattet. Damit ist gesagt: Kraft Gemeingebrauches darf jedermann das Hohe Meer ohne irgendeine Erlaubnis (vom Heimatstaat abgesehen) nach Gutdünken nutzen, sofern die gewählte Nutzung nicht sachwidrig ist (das qualitative Moment des Instituts) oder die gleiche Nutzbarkeit für alle anderen nicht beeinträchtigt (das quantitative Moment - die "Gemeinverträglichkeit").

Demgemäß ist in einem Verdachtsfalle als erstes zu prüfen, ob es nicht - etwa entsprechend Spikes auf Landstraßen - Arten von dem Meer übergebenen Materialien gibt, die geeignet sind, entweder die nutzbare Sache zu vernichten oder für andere Nutzungen durch andere untauglich zu machen. (Vgl. auch den Verf. 131, 165, 174: „irreparable Schäden für die maritime Ökologie“ — etwa durch Einleitung von Quecksilber). Dieses erste, sachliche Element des Gemeingebrauches bedarf der Hervorhebung, weil es entweder übersehen oder jedenfalls mit dem

33 Besprechung von Jochen Ehmer, Der Grundsatz der Freiheit der Meere und das Verbot der Meeresverschmutzung (Berlin 1974) - angezeigt auch von Meyer-Tasch in: "Süddeutsche Zeitung " vom 10. März 1976. Vgl. hierzu ferner Robert A. Shinn. The International Politics of Pollution Control (New York, Praeger) und Jacques Ballenegger, La Pollution en Droit International (Genf 1975). 
zweiten verquickt zu werden pflegt - was sich u. a. daraus erklären mag, daß Übermaß greifbarer ist als Sach- und Bestimmungswidrigkeit.

Obwohl der Verf. immer wieder ganz richtig das Institut "Gemeingebrauch“ seinen Überlegungen zugrundelegt $(32,36,59,89, \ldots .147,159,161,166,169$, 174), wertet er es jedenfalls im Ergebnis nicht aus. Statt unter dem Stichwort „Überschreitung“ (sc der durch Gemeingebrauch gezogenen zweifachen Grenzen) erörtert er die Probleme unter „Mißbrauch“. Hierdurch wird bewirkt, daß eine an sich über den Gemeingebrauch hinausgehende Verschmutzung deswegen noch nicht rechtswidrig ist, daß vielmehr ein weiteres Element hinzutreten muß - der Gebrauch eines Rechtes im fraudem legis. Diese Substitution läuft mindestens im Ergebnis darauf hinaus, daß die relativ sichere Rechtsgrundlage für die Beurteilung von Immissionen ins Meer preisgegeben und an ihre Stelle eine subjektiv gewendete und daher entsprechend unsichere Regel - eben das Verbot des Rechtsmißbrauches - gesetzt wird. Es ist deshalb noch einmal hervorzuheben, daß der Bestimmung der Sache „Meer“ zuwiderlaufende, weil sie vernichtende oder unbrauchbar machende, oder gemeinunverträgliche Nutzungen ipso jure rechtswidrig sind, ohne daß die Frage aufgeworfen werden müßte und dürfte, ob sie mißbräuchlich sind.

Mit Recht erinnert der Verf. immer wieder daran (174, 175, 188, 191), daß „Sachwidrigkeit" und "Gemeinverträglichkeit" sich nach den Umständen richten und daß daher die durch Gemeinbrauch geregelte Freiheit der Meere ebenfalls eine veränderliche Freiheit ist. Die Mitglieder der Völkerrechtsgemeinschaft haben es daher weithin in der Hand, ob der Gemeingebrauch den Nutzungen immer engere Grenzen wird setzen müssen: Die Freiheit wird auch hier nicht eigentlich von „Freiheitsfeinden“ bedroht, die angeblich nichts Schöneres kennen als „Dirigismus" - es sind in erster Linie die Freiheitsfreunde selbst, die es an Umsicht und Verantwortlichkeit fehlen lassen und daher den Gesetzgeber zwingen, durch Normen zu regeln, was Unvernunft, Pleonexie u. a. regelungsbedürftig gemacht haben.

\section{Die Dritte Seerechtskonferenz}

Diesem Thema sind zwei Arbeiten* gewidmet, die ebensowenig wie ihre Vorgängerinnen der Gefahr ausgesetzt sind, durch den berüchtigten „Federstrich des Gesetzgebers" zu Makulatur gemacht zu werden: Denn einmal ist die Konferenz weit davon entfernt, zu einem Ergebnis gelangt zu sein, und selbst - zweitens wenn sie scheitern sollte, würde sie ein so bedeutendes völkerrechtsgeschichtliches Ereignis gewesen sein, daß die ihren Hergang festgehaltenden Arbeiten immer ihren wissenschaftlichen Wert behalten werden.

I. Die erste der beiden Schriften ist weniger eine Vorgeschichte der Konferenz als vielmehr eine umfassende und eingehende Darstellung der Probleme, mit denen sie sich auseinanderzusetzen hat, und deren status causae et controversiae. Man fühlt sich an das Gleichnis vom Senfkorn erinnert: Anstoß zu dieser Konferenz ist die Auffassung gewesen, die Rechtsprobleme des Meeresbodens und seiner Nutzung müßten vor Beginn solcher Nutzung geklärt werden, und die Erkenntnis, sol-

\footnotetext{
* Renate Platzöder, Wolfgang Graf Vitzthum, Zur Neuordnung des Meeresvölkerrechts auf der Dritten Seerechtskonferenz der Vereinten Nationen - Stiftung Wissenschaft und Politik - Ebenhausen 1974. Renate Platzöder, Third United Nations Conference on the Law of the Sea - Documents of the Caracas Session 1974 - Published by Deutscher Verein für Internationales Seerecht und Institut für Internationale Angelegenheiten der Universität Hamburg (Hamburg 1975).
} 
che Klärung sei ohne Regelung aller großen seerechtlichen Probleme, inbesondere aber der seewärtigen Ausdehnung des Festlandsockels, nicht zu bewerkstelligen. Erwachsen ist hieraus eine in jeder Hinsicht umfassende Konferenz: Alle Mitglieder der Völkerfamilie nehmen an ihr teil, und sämtliche Probleme des öffentlichen Seerechts, angefangen von der Ausdehnung des Küstenmeeres, stehen auf ihrer Tagesordnung.

Alledem ist die Aufmerksamkeit dieser wichtigen Schrift gewidmet. Auch sie schenkt als erstes den gerade hier besonders bedeutsamen Rechtstatsachen die gebührende Beachtung (vgl. S. 51: Die Ausdehnung der für den Meeresbergbau abzusteckenden Felder wird um 25000 qm schwanken; S. 234: Die fortschreitende Verminderung der Selbstreinigungskraft der Meere bedroht langfristig die Überlebenschance der Erdbevölkerung). Ferner ist hervorzuheben die tiefdringende Diagnose der zu den Problemen von den einzelnen Staaten und ihren Gruppierungen bezogenen Standpunkte und deren wohlabgewogene Würdigung, wobei abermals auffällt die erstaunliche Übereinstimmung zwischen SU und USA, vor allem in der Frage der Durchfahrt von Kriegsschiffen durch die aus der Ausdehnung des Küstenmeeres auf 12 sm entstehenden neuen Meerengen (27; 32 f;; $40 ; 76 ; 154)$. Nicht unterlassen es auch die Verfasser, die Rechtsfolgen aufzuzeigen, die die Verwirklichung von Vorschlägen mit sich bringen würde: So würde z. B. im Falle der Begründung von Wirtschafts- oder anderer Zonen die Bundesrepublik Deutschland zu einem „zone-locked“-Staat herabsinken (109; 171). Schließlich werden die Forderungen insgesamt im Lichte der großen Entwicklung des Seevölkerrechts gewertet: Das „nationale Hemd“ ist nicht nur den Entwicklungsländern näher als der „internationale Rock“ (161); auch sind die Vorschläge zu einer Internationalisierung des Festlandssockels, der Wirtschaftszone und des Sockelhanges längst begraben (161): Zwar sprechen viele Sachargumente ... gegen "maritimen Nationalismus" und für eine weitgehende Internationalisierung der Meere: „Der Zeitpunkt für eine derartige funktionsgerechte Fortentwicklung des Grundsatzes der Freiheit der Meere dürfte, abgesehen von der küstenfernen Meeresbodenregelung, jedoch noch nicht gekommen sein. Anscheinend muß das Meeresvölkerrecht zunächst eine Phase der Nationalisierung durchlaufen, bevor übernationale Strukturen angestrebt werden können" (173).

Für die deutschen Leser dieser Zeitschrift ist es wichtig, daß die Verfasser gründlich und vor allem auch mit politischem Gespür (dazu auch 142) untersuchen, ob und wie die Interessen der Bundesrepublik Deutschland durch alle geplanten und wahrscheinlichen Neuerungen berührt würden: Das Bild, das sich hieraus ergibt, vermag nur wenig heiter zu stimmen, vor allem was die Ostsee angeht.

II. Mit Recht betonen die Herausgeber der zweiten Schrift, Prof. Dr. Ingo von Münch, Hamburg, als Direktor des Instituts für Internationale Angelegenheiten, und Dr. Walter Hasche, als Präsident des Deutschen Vereins für Internationales Seerecht, daß die von Renate Platzöder erarbeitete Sammlung der Dokumente, die in der Sitzungsperiode Caracas 1974 der Dritten Seerechtskonferenz hervorgetreten sind, ihren Sinn hat und haben wird, gleichgültig ob diese Konferenz eine Kodifikation hervorbringt, oder ob auch hier das Völkerrecht durch die Einzelstaaten und ihren Konsensus gesetzt werden wird. Denn die Seerechtskonferenz ist nach den Haager Konferenzen, die sich mit dem Landkrieg befaßt haben, und den dies fortsetzenden Genfer Konferenzen von 1949 der bedeutenste internatio- 
nale Versuch, der sich die Kodifikation einer umfassenden Völkerrechtsmaterie zum Ziele gesetzt hat. Ob gelungen oder gescheitert - ein solcher Versuch wird nicht nur deswegen erheblich sein, um die Ursachen $\mathrm{zu}$ erforschen, warum er geglückt oder mißlungen ist. Man ist daher den Veranstaltern dieser wissenschaftlichen Leistung wie vor allem ihrer Vollbringerin zu lebhaftem Dank dafür verpflichtet, daß sie diesen völkerrechtlich in jeder Hinsicht ungemein wichtigen Vorgang dokumentarisch festgehalten und dadurch dessen spätere Erforschbarkeit gesichert haben.

\section{Adekunle Ajala}

\section{Pan-Africanism: Evolution, Progress and Prospects}

André Deutsch, London 1973, pp. XIII+442.

Mr. Ajala has written a readable and informative book which should be read by those seriously concerned or worried by the developments in Africa, for PanAfricanism, whether motivated by political and economic self-defence or by reaction to centuries of colonialist and racialist aggression, is a force to reckon with. The first part of the book is an interesting account of the evolution of the Pan-African movement. Between the two world wars, the movement was centred in the United States of America where the radical, anti-racist racialism of Marcus Garvey and the Universal Negro Improvement Association, and the "moderate", bourgeois DuBois and the N. A. A. C. P. (National Associaion for the Advancement of Colored People) symbolised the opposing currents in Pan-Africanism which are still with us to-day. The contribution of Africans at this time was slight. After 1945 the centre of gravity of Pan-Africanism moved to Africa. Nkrumah, Kenyatta, George Padmore (West Indian) and R. Makonnen (West Indian) were the dominant figures of this period. The first Conference of Independent African States, held in Accra from 15 to 22 April, 1958 marked the definite establishment of the Pan-African movement in Africa. The 1960 avalanche of independence added to the strength of the movement on African soil. The O. A. U. (Organization of African Unity), created on 25th May, 1963 gave a more concrete structure to Pan-Africanism, even though it was a compromise organization, intended to reconcile the radical Casablanca Group of African states with the moderate Monrovia Group. Mr. Ajala ends this part of the book with a review of the ideas of the men who influenced the course of Pan-Africanism, classifying them as "torchbearers" (Marcus Garvey, William E. B. DuBois), "progressives" (Kwame Nkrumah and Sekou Touré) and "moderates" (Leopold Senghor, Felix Houphouet-Boigny, Emperor Haile Selassie and Tafawa Balewa). This historical introduction, which I found extremely useful, shows that the obstacles in the way of Pan-Africanism have a long history behind them. Many readers will no doubt appreciate the present significance of this statement from Marcus Garvey: "In the fight to reach the top the oppressed have always been encumbered by the traitors of their own race, made up of those of little faith and those who are generally susceptible to bribery for the selling out of the rights of their own people."(p.91)

The second part of the book is devoted to the examination of the achievements and failures of the movement. Mr. Ajala correctly states that the foremost achievement of Pan-Africanism before 1963 was creating the feeling of African- 